\title{
Determinants of visual acuity outcomes in eyes with neovascular AMD treated with anti-VEGF agents: an instrumental variable analysis of the AURA study
}

Eye (2017) 31, 166; doi:10.1038/eye.2016.276

Correction to: Eye (2016) 30, 1063-1071; doi:10.1038/eye.2016.90; published online 20 May 2016

Since the publication of this article, the authors would like to correct a mistake in the heading of Table 2. The lower $95 \%$ confidence interval (CI) and upper $95 \%$ CI should be replaced with the
FG Holz, R Tadayoni, S Beatty, AR Berger, MG Cereda, P Hykin, G Staurenghi, K Wittrup-Jensen, J Nilsson, K Kim and S Sivaprasad
Year 2 coefficient and $P$-value, respectively. Accordingly, the first column should be replaced with Year 1 coefficient and $P$-value (as shown). The coefficients for Year 1 are quoted in the text, but this does not change the interpretation of the findings.

The authors would like to apologise for this error.

Table 2 Correlation between number of ranibizumab injections and candidate variables (all countries; over 2 years)

\begin{tabular}{|c|c|c|c|c|}
\hline \multirow[t]{2}{*}{ Parameters } & \multicolumn{2}{|c|}{ Year 1} & \multicolumn{2}{|c|}{ Year 2} \\
\hline & Coefficient ${ }^{\mathrm{a}}$ & P-value & Coefficient $^{\mathrm{a}}$ & P-value \\
\hline Age at start of therapy & -0.0289 & 0.1141 & -0.0378 & 0.056 \\
\hline Sex & 0.0171 & 0.384 & -0.0126 & 0.5193 \\
\hline Health insurance (public) & -0.028 & 0.1544 & -0.053 & 0.0071 \\
\hline Reimbursement type (national standards) & 0.1297 & $<0.001$ & 0.1524 & $<0.001$ \\
\hline Medical history (prior disease) & -0.0268 & 0.1711 & -0.0354 & 0.0703 \\
\hline Medical history (concomitant disease) & -0.0003 & 0.9866 & 0.018 & 0.3595 \\
\hline Medical history (ocular disease) & -0.0246 & 0.2083 & -0.0331 & 0.0905 \\
\hline Baseline VAS & -0.0088 & 0.6758 & 0.0424 & 0.0444 \\
\hline Treatment duration & 0.5645 & $<0.001$ & 0.7389 & $<0.001$ \\
\hline Switch to other treatments & 0.0275 & 0.1599 & 0.0632 & 0.0012 \\
\hline Baseline presence of retinal breaks ${ }^{\mathrm{b}}$ & NA & NA & NA & NA \\
\hline Baseline presence of pigment epithelial detachment & 0.0821 & 0.0038 & 0.1163 & $<0.001$ \\
\hline Number of ophthalmoscopies & 0.2499 & $<0.001$ & 0.3425 & $<0.001$ \\
\hline Optical coherence tomography, $n$ & 0.3777 & $<0.001$ & 0.4955 & $<0.001$ \\
\hline Fluorescein angiography, $n$ & 0.0509 & 0.0093 & 0.1154 & $<0.001$ \\
\hline ICGA, $n$ & -0.0341 & 0.0814 & 0.0302 & 0.1228 \\
\hline Ophthalmoscopies/optical coherence tomography, $n$ & 0.3608 & $<0.001$ & 0.4673 & $<0.001$ \\
\hline Number of monitoring visits & -0.1349 & $<0.001$ & 0.0239 & 0.2224 \\
\hline
\end{tabular}

Abbreviations: ICGA, indocyanine green angiography; NA, not available; VAS, visual acuity score. ${ }^{\text {PPearson's correlation }}$

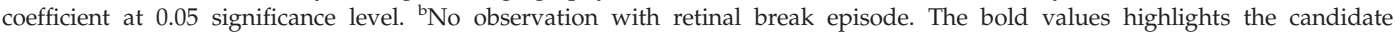
instrumental variables. 